\title{
An Engineered Toolkit for Precise Spatiotemporal Regulation of Protein Nucleo-cytoplasmic Distribution Derived from Programmable RfA1 Sequence
}

\author{
Junyi Song *,+, Chuanyang Liu ', Baoshan Li, Liangcheng Liu, Ling Zeng, Zonghuang Ye, Wenjian Wu, Zhu \\ Lingyun * and Biru $\mathrm{Hu}$ *
}

College of Liberal arts Science, National University of Defense Technology; songjunyi@nudt.edu.cn (J.S.); liuchuanyang13@nudt.edu.cn (C.L.); 1678213718@qq.com (B.L.);290343303@qq.com (L.L.); zengling14@nudt.edu.cn (L.Z.); yezonghuang15@nudt.edu.cn (Z.Y.); wjwu67@nudt.edu.cn (W.W.)

* Correspondence: songjunyi@nudt.edu.cn (J.S.); lingyunzhu@nudt.edu.cn (Z.L.); hubiru08@nudt.edu.cn (B.H.); Tel.: +86 0731-84501815

+ These authors contributed equally to this work.

\begin{abstract}
Proteins, as gifts from nature, provide structure, sequence, and function templates for designing biomaterials. Here, we reported an engineered toolkit derived from a natural block copolymer, RfA1. RfA1 is composed of positively charged polyelectrolyte linker regions interspersed with highly conserved polyampholyte motifs. These linkers and motifs are constructional fragments and ready-to-use building blocks for synthetic design and construction. One functional and editable feature of RfA1 derivatives is their preferential distribution to cytoplasm or nucleoplasm, in a fragment-replication-determined manner. Based on this property, a prices spatiotemporal Tet-on demo was established, which effectively transports cargo peptides into nuclei at selective time points. Moreover, the functional homogeneities of either motifs or linkers were also verified, making them standardized building blocks for synthetic biology. In summary, this study provides a modularized, orthotropic and well-characterized toolkit for precise and spatiotemporal regulation of protein nucleocytoplasmic localization.
\end{abstract}

Keywords: RfA1; engineered peptides; spatiotemporal tunable; cyto/nucleoplasmic location

\section{Introduction}

Due to the richness of the amino acid arrangements and combinations available, peptides and their derivatives are highly versatile structural, and functional building blocks [1-3]. On one hand, artificial designed peptides are able to generate various architectures (including fibres, tapes, tubes, sheets and spheres [3]) in vitro, which demonstrates considerable potential for carrier-mediated drug delivery, tissue engineering, antimicrobial agents, imaging tools, energy storage, biomineralization, and membrane protein stabilization [4]. On the other hand, peptides and relative derivatives have been developed as effective "navigation system" to selectively target to organelles, e.g., endoplasmic reticulum[5,6], mitocondria $[7,8]$, or nucleus $[9,10]$. Since exact localization of proteins is required to fulfill their biological functions[11], transportation of functional proteins or peptides to orientated intracellular localization is a prerequisite to intensify their functions in application areas $[12,13]$, or to properly study their mechanism in basic research fields $[14,15]$. Bidirectional transportation of proteins in or out nuclear membrane is such a dogmatic example. Inwards, proteins are translated in the cytoplasm, but many need to be transport into the nucleus to perform their functions [16]. Outwards, RNA-protein complex need to be dynamically exported out from the nucleus into cytoplasm $[17,18]$. 
In this point, molecular tools able to quantitatively regulate the entry and exit of target proteins into and out of nucleus are of great value, which bring in various novel applications in synthetic and cell biology fields [9,18,19].

Genetical construction of nuclear localization signal (NLS) sequences into cargo proteins is the most routine approach, which has successfully transport functional proteins $[9,10]$, genome-editing elements $[20,21]$, and transcriptional circuits $[22,23]$ into nucleus. Similar but in the opposite direction, the utilization of NES (nuclear export signal) allows the translocation of molecular components out of nucleus [9,24]. By embedding light-activated domains into NLS or NES, the orientated trafficking can further present light-responsive properties [24-26]. Besides, various NLS sequences have been used as delivery agents to enhance cellular uptaking and nuclear targeting of plasmid DNA [27-29], or other functional nano cargoes [30-32]. In this regard, identification and engineering of guiding sequences such as NLSs and NESs will bring in prosperous advanced applications.

Here, we report a novel guiding system based on programmable RfA1 sequences, which is able to temporal regulate the nucleo-cytoplasmic localization of peptide cargoes. RfA1 is a natural block copolymer composed of positively charged polyelectrolyte linker regions (reflectin linkers, RLs) interspersed with highly conserved polyampholyte segments (reflectin motifs, RMs) [33,34]. In this system, RLs and RMs are well-prepared and ready-to-use building blocks for the construction of synthetic functional molecules. As the first step, four native reflectins RfA1, RfA2, RfB1 and RfC were introduced into HEK-293T cells, and were found to preferentially enrich in nuclei or cytoplasm. Considering their sequence differences, the repetition of conserved motifs is likely to in charge of the selective intracellular localization. To verify this hypothesis, RfA1 and its derivatives were designed and engineered into cells. RLNto1, RLNto2 and RLNto3 were found to effectively transport GFP (as a cargo molecule) into nucleus, while RLNto5 causes an exclusively cytoplasmic enrichment of GFP outside the nucleus. This strict intracellular localization of RfA1 derivatives confirms the motif-repetition-dependent hypothesis, and suggests them as editable guiding tags to transport molecular cargoes to selective regions. Subsequently, by integrating Tet-On system $[35,36]$ with RLNto2, the precise nuclear enrichment was then temporal regulated by doxycycline administration. In this case, Tet-On components works as the launch button, while RfA1 derived sequences are guided missiles which carry molecular cargos to prefixed targets. At last, to verify the functional homogeneity of RMs and RLs during subcellular localization, genes of two de novo designed peptides $\mathrm{RM}_{\mathrm{N}}+\mathrm{RM}_{1}{ }^{*} 5$ and $\mathrm{RM}_{1}{ }^{*} 3+\mathrm{RL}_{2} * 2$ were synthesized. For these two peptides, the subtle differences among motifs or linkers were eliminated. In this assay, the distribution of these two de novo peptides is exactly similar to comparable RfA1 derivatives, indicating that these peptide building blocks can be unified and standardized.

Briefly, this study identified series of building blocks from reflectins amino acid sequences. Reorganization of these building blocks leads to an exclusive cytoplasmic or nucleoplasmic enrichment of ligated molecular cargos (e.g., GFP), which quantificationally determined by times of RMs and RLs repetition. Combined with other synthetic biologybased tools, this programmable RfA1 derived strategy can be further upgraded as a spatiotemporal controllable toolkit to realize precise intracellular delivery.

\section{Results}

2.1. Subcellular localization of reflectin proteins and deconstruction of RfA1 sequence

Two types of patterned $\sim 25$ amino acid methionine-rich motifs are reported in reflectin sequence: the N-terminal motif (RMN) [MEPMSRM(T/S)MDF(H/Q)$\mathrm{GR}(\mathrm{Y} / \mathrm{L})(\mathrm{I} / \mathrm{M}) \mathrm{DS}(\mathrm{M} / \mathrm{Q})(\mathrm{G} / \mathrm{D}) \mathrm{R}(\mathrm{I} / \mathrm{M}) \mathrm{VDP}(\mathrm{R} / \mathrm{G})]$ and a series of conserved reflectin motifs (RMs) [M/FD(X)5MD(X)5MDX3/4][37] (Fig.1a). The N-terminal region is more evolutionarily conserved across species and reflectin isoforms than the canonical $\mathrm{RM}_{\mathrm{N}}[38]$, while almost all " $\mathrm{X}$ " sites are populated largely by one specific residue with minor alternative 
residues usually represented in only one or a few reflectin motifs in the entire known library [39].

At present, studies are mostly focused on their self-assemly properties in vitro $[33,40,41]$. The dynamic reflectin assembly properties have already inspired the development of various next-generation tunable photonic [42-44] and electronic platforms and devices [45-47]. However, our knowledge about their performance in cells is relatively limited.

To investigate their characteristics in cell, four reflectin proteins were constructed into pEGFP-C1 vectors and transfected into human embryonic kidney (HEK) 293T cells. Compared with cells transfected by no-load pEGFP-C1, all these four reflectins tends to form protein condensates or spherical droplets in cell. The formation of proteinaceous condensates is consistent with computational analysis, which suggests reflectins ass potential intrinsically disordered protein to execute phase separation [33]. More significantly, the RfA1 condensates exclusively distribute in cytoplasm, while RfA2, RfB1 and RfC droplets were highly enriched in nuclei (Fig. 1b).

Viewing from their sequence composition, the most obvious commonality is the existence of RMs, while the most significant difference is the times of RMs and RLs repetitions.

To explore the role of conserved RMs during the protein condensation and selective localization, we cloned the six RMs of RfA1 ( $R M_{N}$, and $R M_{1,2,3,4,5}$, primers in Supplement Table. 1) and introduced them into cells, respectively. Results shown that all individual RMs distribute freely in both cytoplasm and nucleoplasm, with no phase separation tendency (Fig. 1c). Therefore, other than conserved amino acid composition, the cytoplasmic enrichment and phase separation of reflectins should be driven by its segmented sequence structure. That is to say, the formation of reflectin condensates or droplets follows a canonical rule: multivalent interaction between repeated motifs and flexible likers is essential for the biomolecular liquid-liquid phase separation $[48,49]$.

a

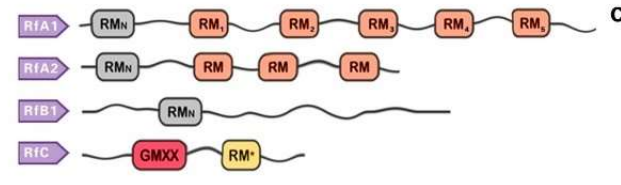

b

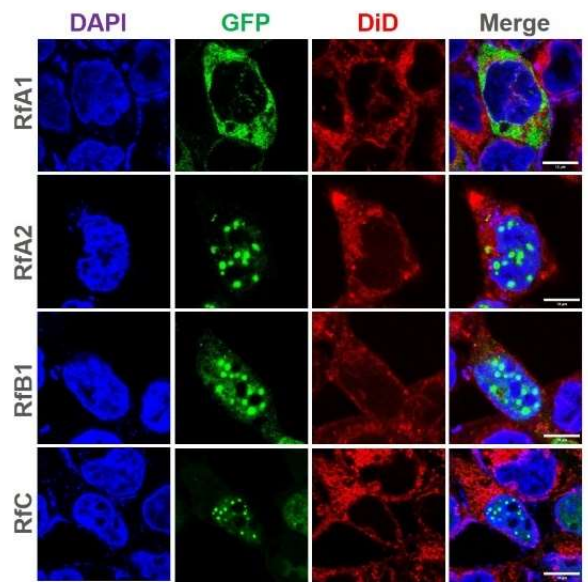

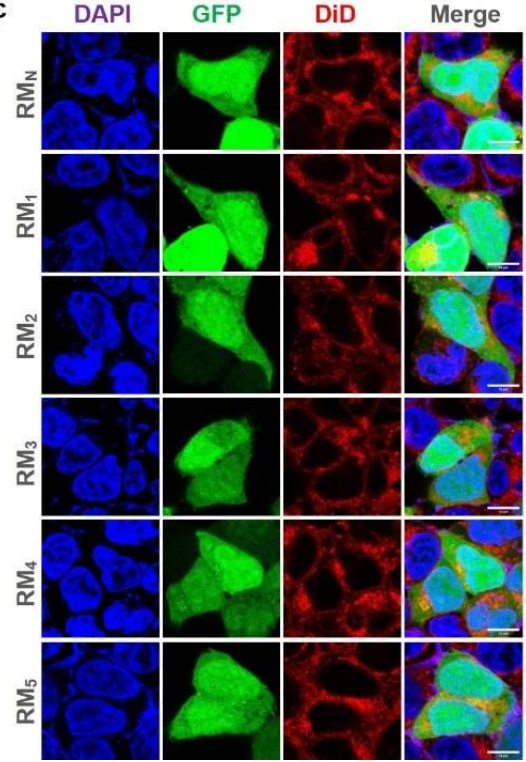

Figure 1. Selective Intracellular Localization of Reflectins and Free-Distribution of Single Motifs. a Schematics of reflectin proteins sequences. Conserved Reflectin Motifs (RMN and repeated RM1-5) are designated by boxes, while reflectin linkers (RLs) are lines. b,c Fluorescence microscopy images of transfected HEK-293T cells stained with DAPI, DiD, while reflectins and variants are visualized by tandem EGFP, Scalebar $=10 \mu \mathrm{m}$. 


\subsection{Reconstruction of block amino acid sequence}

Considering the composition necessity of an intrinsically disorder protein, five pairs of primers were designed to clone the DNA sequences from RfA1 gene (primers in Supplement Table. 2), to gradually extend peptide sequences and restore their segmented structure. The PCR products responsible for coding RLNto1, RLNto2, RLNto3, RLNto4 and RLNto5 are subsequently ligated to the vector pEGFP-C1. For cells expressed with RLNto1, RLNto2, RLNto3, the common characteristics is their enrichment in nuclei. This is totally different from the cytoplasmic-localization-preference of RfA1 or free-distribution of single RMs, but quite similar to simpler reflectins (RfA2, RfB1, RfC). Besides, RLNto2, RLNto3 not only enriched in cytoplasm, but also began to phase out from the crowded cellular milieu. As sharp contrasts, longer RLNto4 and RLNto5 started to escape from the nuclei and form condensates in cytoplasm, extremely resemble their "ancestor" RfA1.

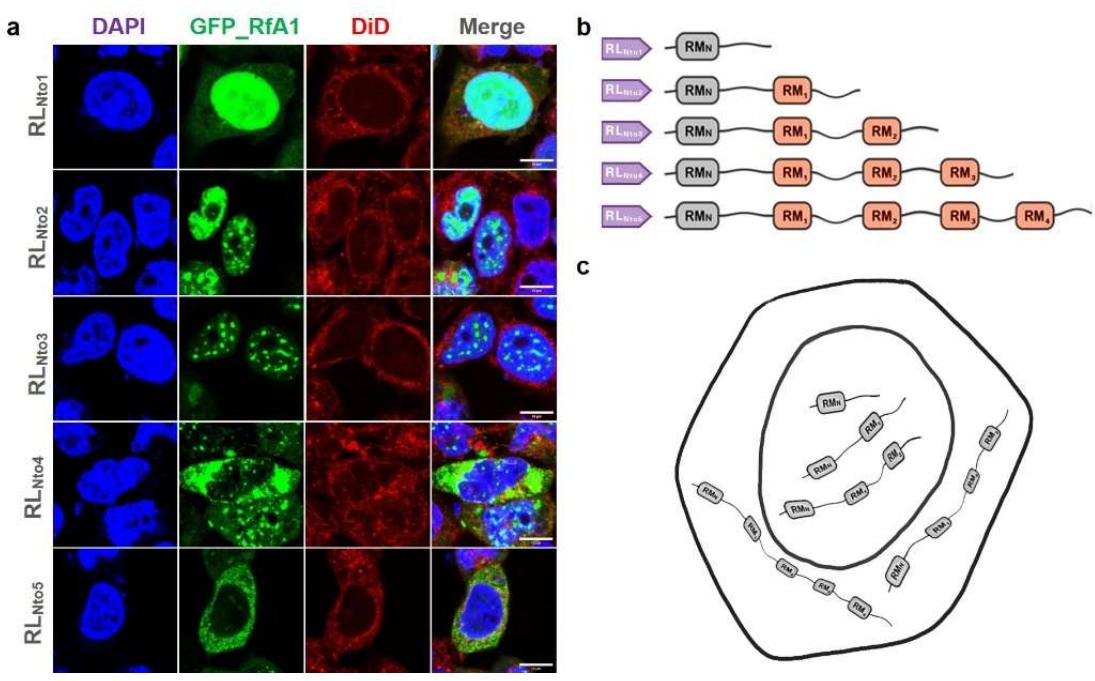

Figure 2. Recurrence of phase separation and cyto-/nucleo-localization preferences of RfA1-derived peptides in fixed HEK-293T cells. a Nuclei and membrane are stained with DAPI (blue) and DiD (red), RfA1-derived peptides are indicated by tandem EGFP (green), Scale bars $10 \mu \mathrm{m}$. b Schematics of RfA1 derivatives. c Illustration of subcellular localization of RLNto1, RLNto2, RLNto3, RLNto4 and RLNto5.

In 2017, Guan et.al. reported that reflectin motifs may be traced to a 24-bp transposon-like DNA fragment from the symbiotic bioluminescent bacterium Vibrio fischeri [50]. Afterward, million years of self-replication and translocation of that transposon leads to the formation of prosperous reflectin family. Here, the distinguished characteristics of short RfA1 truncations and their comparable behaviors with RfA2, RfB1 and RfC is a strong clue to support Guan's evolution hypothesis.

Otherwise, seeing from an application perspective, the distinct repetition-dependent nucleocytoplasmic localization preference strongly recommends RfA1 as a programmable and editable intracellular guiding/navigation tag. If we take GFP as a molecular cargo, reflectin derivatives can be regarded as intelligent vehicles to transport cargoes to preselected destinations (cytoplasm or nucleoplasm). Based on this consideration, we began to test the application potentials of RfA1 variants as synthetic biology tools. As the shortest peptide targets to nuclei and phase out from the nucleoplasm, RLNto2 was used in the following studies and regarded as a guiding tag.

\subsection{Dose-dependent and Time-scale Expression of RLNto2}

For dose-dependent assays, the final concentrations of recombinant pEGFP-C1-RLNto2 plasmids were adjusted from 0.2 to $1.2 \mathrm{ug} / \mathrm{mL}$ (Fig. 3a). As the plasmid concentration went up, numbers of transfected cells and corresponding Statistical Areas increased gradually (Fig. 3b). However, the GFP_RLNto2 expression level (indicated as Average Intensity) of 
each cell reached its upper limit when the plasmid concentration was merely $0.4 \mathrm{ug} / \mathrm{mL}$. That is to say, the pEGFP-C1-RLNto2 concentration mainly influences transfection efficiency, with minor effects on the quantity of plasmid uptook by each cell or the expression level of each cell. Accordingly, the upward trend of gross production of GFP_RLNto2 (Total Intensity, Fig. 3d) is more relevant and similar to the increasing number of transfected cells (indicated by Statistical Area).

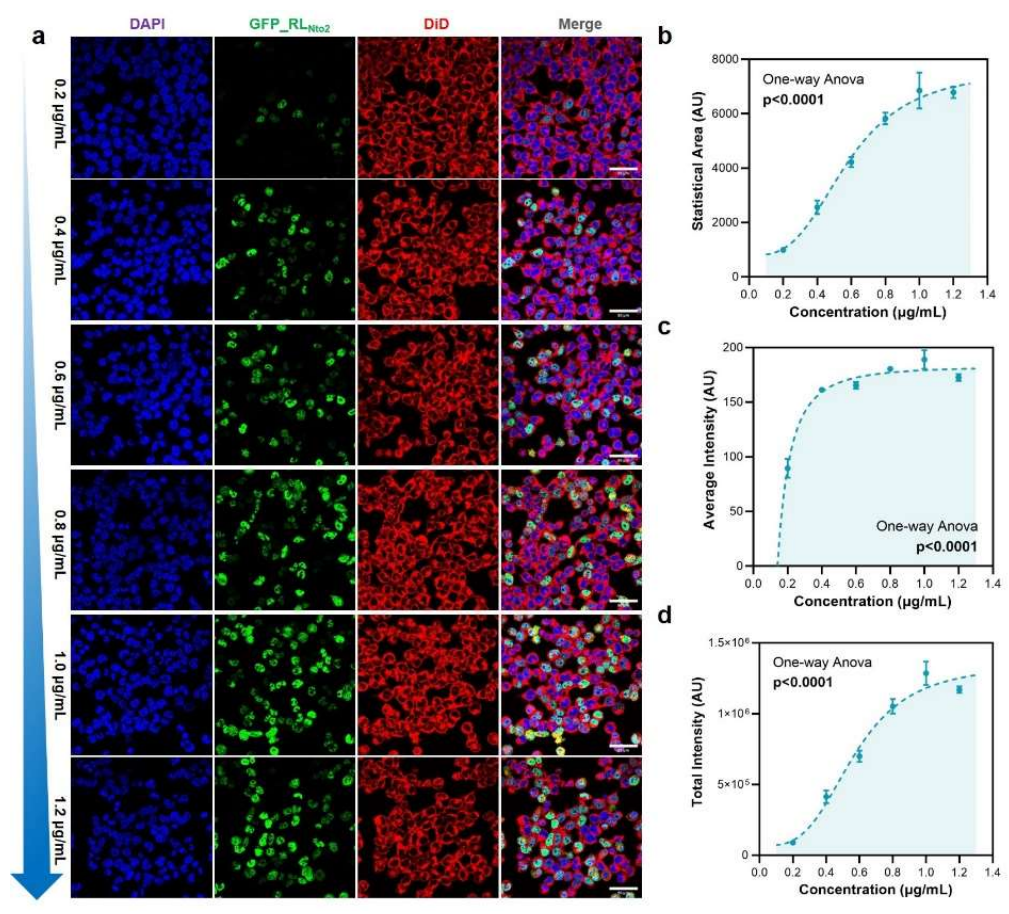

Figure 3. Dose-dependent Expression of RLNto2. a. Confocal observation of HEK-29ET cells transfected with different plasmid concentrations. Nuclei and membrane are stained with DAPI (blue) and DiD (red), RLNto2 is indicated by tandem EGFP (green), Scale bars $50 \mu \mathrm{m}$. b. Statistical Area, refers to the area indicated by GFP green fluorescent signal; c. Average Intensity, refers to the average fluorescent intensity and indicates the expression level; $\mathbf{d}$. Total Intensity, represents the gross expression of GFP_RLNto2. 

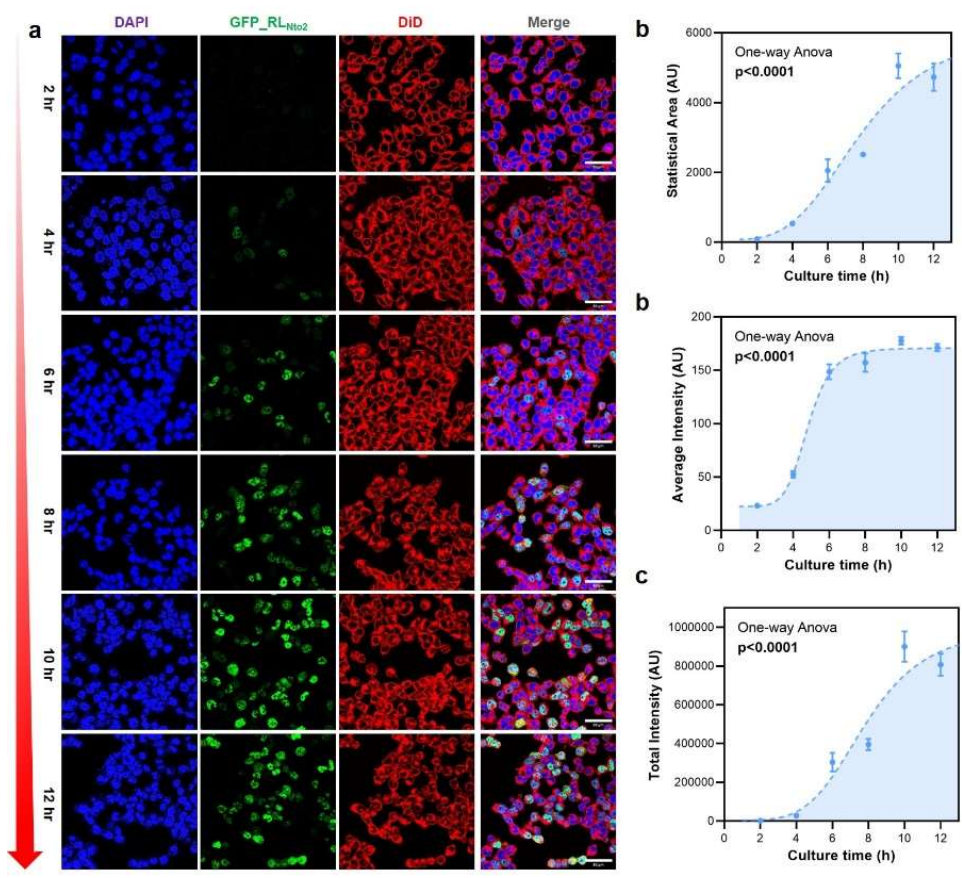

Figure 4. Time-scale Expression of RLNto2. a. Confocal observation of HEK-29ET cells transfected with $1 \mu \mathrm{g} / \mathrm{mL}$ plasmids, at different time points. Nuclei and membrane are stained with DAPI (blue) and DiD (red), RLNto2 is indicated by tandem EGFP (green), Scale bars $50 \mu \mathrm{m}$. b. Statistical Area, refers to the area indicated by GFP green fluorescent signal; c. Average Intensity, refers to the average fluorescent intensity and indicates the expression level; d. Total Intensity, represents the gross expression of GFP_RLNto2.

Similarly, the time-scale expression of RLNto2 was also surveyed. When the confluence reached $\sim 70 \%$, cells were fed with pEGFP-C1-RLNto2 plasmids at concentration of $1 \mu \mathrm{g} / \mathrm{mL}$. For each batch, 6 dishes of cells were used, and took for observation one by one for every $2 \mathrm{~h}$ (Fig. 4a). From a time-span of 2 to $10 \mathrm{~h}$, the transfection efficiency (Fig. $4 \mathrm{~b}$ ) gradually increased, resulting in the steadily rising of GFP-RLNto2 gross production (Fig.5d). After 10 $\mathrm{h}$, the expression level tended to be stable. For each transfected cell, expression level reached the peak around 6 to $8 \mathrm{hr}$ (Fig. 4c).

\subsection{A Doxycycline-induced Teton system integrated with $R L_{N \text { to2 }}$}

To further exhibit its application potential as a synthetic biology component, RLNto2 was constructed into Teton plasmids, which can be easily switched on or off by doxycycline (dox) administration (Fig. $5 \mathrm{a}$ ). When the cell confluence reached $\sim 30 \%$, transfection was conducted according to a standard protocol. Transfection efficiency was checked by fluorescent images after $24 \mathrm{~h}$. Cells were then treated with concentration-gradient dox and cultivated for another $24 \mathrm{~h}$. Afterwards, the nuclei-targeted expression of RLNto2 was observed (Fig. 5b) by confocal system. Obviously, the expression level of RLNto2 was enhanced synchronously with the dox concentration gradient. This implies the successful activate of this Teton system and its controllability based on the dox dose-dependent performance. 
a b

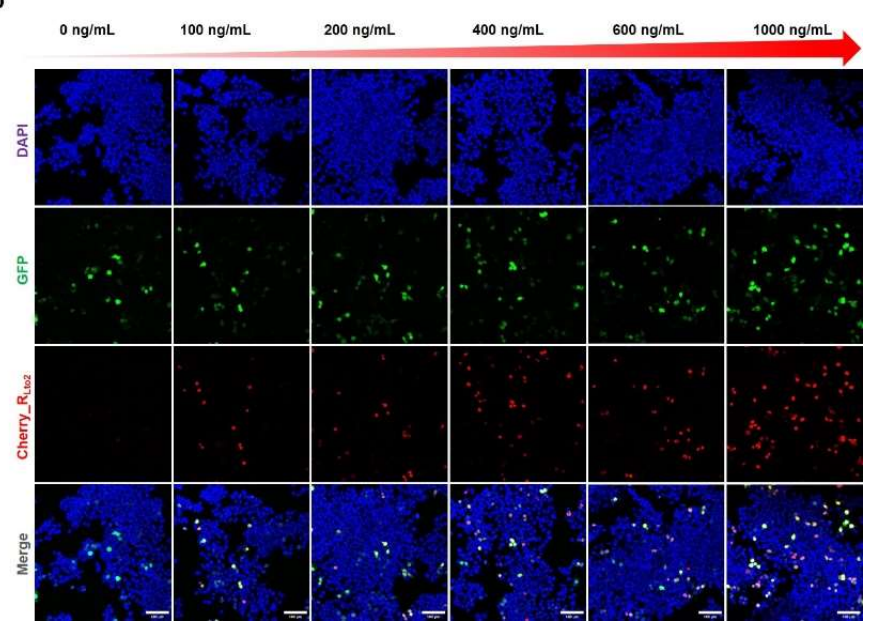

Figure 5. A dose-dependent nuclei-targeted TetOn-RLNto2 system mediated by doxycycline. a schematic diagram of TetOn-Rfs system. b Activation of TetOn-Rfs system. Transfected cells were indicated by GFP (green), the localization of RLNto2 was labeled by tandem Cherry (Red), Scale bars $100 \mu \mathrm{m}$.

Foreseeable, if reporter gene $m$ Cherry was replaced by other functional or therapeutical peptides, this TetOn-Rfs system is able to precisely transport the molecular cargos into nuclei, to amplify their biological effects. Besides, if certain components prefer to fulfil their functions in cytoplasm, RLNto2 could be replaced by RLNto4 or RLNto5. In this point, the programmable RfA1 sequences provides an editable and selectable engineered toolkit, which can be used to regulate precise spatiotemporal localization of proteins in cells.

\subsection{Verification of standardisation of reflectin-derived building blocks}

Truncated peptides discussed previously are regionally $100 \%$ match to the original RfA1 amino acid sequence. To further testify their potential as standard elements for synthetic biology applications, two de novo peptide sequences are designed: a $\mathrm{RM}_{\mathrm{N}}+\mathrm{RM}_{1}{ }^{*} 5$ peptide, with all $\mathrm{RM}_{1 \sim 5}$ replaced by a unified $\mathrm{RM}_{1}$; $\mathrm{a}_{\mathrm{R}} \mathrm{R}_{1}{ }_{3}+\mathrm{RL}_{2}{ }^{*} 2$ peptide, while $\mathrm{RL}_{2}$ refers to the linker between $\mathrm{RM}_{1}$ and $\mathrm{RM}_{2}$ (sequence information refers to Supplementary Table. 3, sketches of artificial peptides shown in Fig6. b,d). Highly consistent with full-length RfA1 in Fig. 1, RMN+RM1 ${ }^{*} 5$ triggers a remarkable phase separation in cytoplasm. In the meanwhile, $\mathrm{RM}_{1}{ }^{*} 3+\mathrm{RL}_{2}{ }^{*} 2$ molecules are somehow transported into nuclei and underwent phase separation afterwards (Fig. 6c). These results suggest that the building blocks or functional components derived from reflectin amino acid sequences can be standardized without losing their intracellular localization preferences, which favors their application in synthetic biology fields.

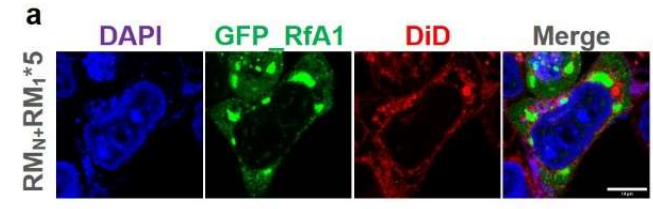

b

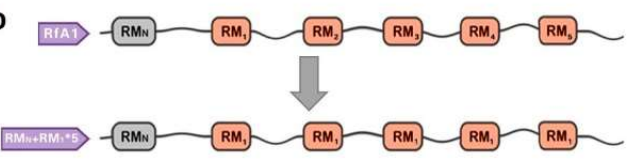

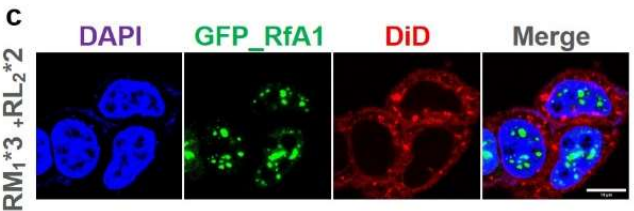

d

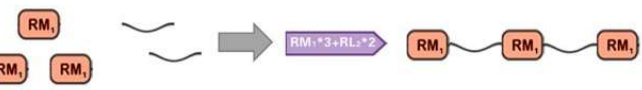

Figure 6. a,c Fluorescence images of fixed HEK-293T transfected with pEGFP-C1-(RMN+RM1 $\left.{ }^{*} 5\right)$ and pEGFP-C1$\left(\mathrm{RM}_{1} * 3+\mathrm{RL}_{2}{ }^{*}\right)$, respectively. Nucleus and cytomembrane are stained with DAPI and DiD. $\mathbf{b}, \mathbf{d}$ Schematics of $R M_{N}+R M_{1} * 5$ and $\mathrm{RM}_{1}{ }^{*} 3+\mathrm{RL}_{2} * 2$ sequences. 


\section{Discussions}

As essential biomacromolecules, the exact localization of proteins is required for organelles to work correctly [11]. Proteins are translated in the cytoplasm, but many need to be transport into the nucleus to perform their functions [16]. On the other hand, the dynamic export of RNA-protein complex out from nucleus is also a central but not fully understood process for molecular biology $[17,18]$. Hence, molecular tools which are able to qualitatively and quantitatively regulate the entry and exit of target proteins into and out of nucleus bring in various novel applications in synthetic and cell biology $[9,18,19]$.

Here, a novel guiding toolkit derived from reflectin amino acid sequence is developed.

Being curious about their intracellular functions and properties, genes of four native reflectins (RfA1, RfA2, RfB1 and RfC) were introduced into HKE-293T cells. Interestingly, though share some compositional homologies, the localization preferences of reflectins were distinguishable: RfA1 exclusively located in cytoplasm, while RfA2, RfB1 and RfC were highly enriched in nucleoplasm. According to Guan's report, reflectin genes in cephalopods come from a transposon in symbiotic Vibrio fischeri [50]. In this point of view, shorter and simpler RfC should originally emerge as the ancestor molecular in nucleus. Then longer reflectins began to be assembled and to escape from the nucleus into cytoplasm.

In the meanwhile, from a biosynthetic application perspective, reflectin sequences provide programmable building blocks to guide cargo molecules and achieve selective orientation. Take RfA1 as the initial template, by cutting RMs off one by one from RfA1 amino acid sequence via gene engineering, longer RfA1 derivatives with more RMs repetitions tends to stay in cytoplasm, while shorter RfA1 truncates started to enter nuclei. Moreover, we integrated the RfA1 derived guiding peptides with Tet-On system. In this demo, Tet-On elements work as a trigger, allowing precise activation at scheduled time point under the regulation of doxycycline; while RfA1 derivatives RLNto2 works as precision-guided missiles, which effectively carry the molecule cargo (e.g., mCherry) into nuclei.

At last, by replacing $\mathrm{RM}_{2,3,4,5}$ in RfA1 with unified RM1 or de novo designing an artificial peptide "RM1 ${ }^{*} 3+\mathrm{RL}_{2}{ }^{*} 2^{\text {", }}$, the functional homogeneity of RMs and RLs were verified. It means that, for cyto- or nucleo-targeting, guiding sequences derived from RfA1 amino acid sequence can be modified as unified and standardized building blocks. By combining with other responsive synthetic biology components, this RfA1-derived strategy and standadized building blocks can be further programmed and developed as versatile and spatiotemporal controllable toolkit.

\section{Materials and Methods}

\subsection{Construction of recombinant $p E G F P-C 1$ vectors}

Nucleotide sequence of D. (Loligo) pealeii reflectin A1 (RfA1) (Genbank: ACZ57764.1) and D. (Loligo) Opalescens reflectin C (Genbank: AIN36559.1) were optimized for human cell expression, then synthesized and sequencing-identified by Sangon Biotech ${ }^{\circledR}$ (Shanghai, China) Primers (F-GAATTCTAT GAATAGATATTTGAATAGACA; RGGATCCATACATATGATAATCATAATA ATTT) were designed to introduce EcoR I and BamH I cutting sites, so the modified RfA1 CDS can be constructed into pEGFP-C1 via a standard restriction enzyme cloning process. As for truncated RfA1 derivatives, six pairs of primers were coupled used. Take this for example, if $\mathrm{RM}_{\mathrm{N}}-\mathrm{F}$ and $\mathrm{RM}_{3}-\mathrm{R}$ primers were selected, then a nucleotide sequence responsible for the coding of $R M_{N}-R_{1}-R_{1}-$ $\mathrm{RL}_{2}-\mathrm{RM}_{2}-\mathrm{RL}_{3}-\mathrm{RM}_{3}$ (which is simplified as RMNto3 in this paper) will be obtained after PCR. In the meanwhile, 5' GCATGGACGAGCTGTACAAG 3' and 5' TTATGATCAGTTATCTAGAT 3' were added to those F-primers and R-primers respectively during primers synthesis, which enables sequences to be ligated to pEGFP-C1 by Ready-to-Use Seamless Cloning Kit from Sangon Biotech ${ }^{\circledR}$ (Shanghai, China). 


\subsection{Growth and transfection of human cells}

HEK-293T cells (ATCC ${ }^{\circledR}$, CRL-3216 $^{\mathrm{TM}}$ ) were cultured on plastic dishes in Dulbecco's Modified Eagle Medium (DMEM, Gibco ${ }^{\mathrm{TM}}$ ) supplemented with 10\% fetal bovine serum (FBS, Gibco ${ }^{\mathrm{TM}}$ ) at a temperature of $37^{\circ} \mathrm{C}$ and under $5 \% \mathrm{CO}_{2}$. One day before transfection, cells were seeded at $\sim 33 \%$ of the confluent density for the glass bottom dishes from Cellvis (California, USA), and grown for another $24 \mathrm{~h}$. Then transfection mixture containing Lipofectamine 3000 (Thermo Scientific) and recombinant vectors was added to the medium, incubating for $\sim 16$ to $\sim 24 \mathrm{~h}$. For CCK- 8 tests, $1 \times 10^{4}$ cells were seeded into each hole of 96-well plates one day before transfection, then transfected with recombinant vectors and incubated for another $24 \mathrm{~h}$. After that, $10 \mu \mathrm{l}$ of CCK-8 solution will be added into wells for a $\sim 2$ to $\sim 4 \mathrm{~h}$ chromogenic reaction. OD 450 was detected by Multiskan FC (Thermo Scientific).

\subsection{Fluorescence microscopy of stained cells}

Transfected HEK-293T cells grown in Cellvis plastic dishes were firstly fixed with $4 \%$ paraformaldehyde at room temperature for 30 mins, then stained with DiD or ActinRed (diluted in $0.5 \%$ triton $X-100$ PBS) for $~ 30$ mins after PBS rinses. After washing off the fluorescent dye with PBS, fixed cells will be embedded in DAPI-Fluoromount (Beyotime, Shanghai, China) and characterized with a Leica TCS SP8 imaging system in fluorescence imaging mode. The resulting images were analyzed with ImageJ (Java 1.8.0_172/1.52b) [51].

Supplementary Materials: The following supporting information can be downloaded at: www.mdpi.com/xxx/s1, Table S1, S2, S3.

Author Contributions: J.S. and C.L designed and performed experiments, analyzed data, and wrote the manuscript. L.Z. assisted in directing graduate students to carry out experiments. B.L. and L.L. performed experiments. Z.Y. analyzed data. W.W., L.Z. and B.H. designed experiments and wrote the manuscript. All authors have read and agreed to the published version of the manuscript.

Funding: This research was funded by National Natural Science Foundation of China, grant number 31971291; Natural Science Foundation of Hunan Province, grant number S2020JJQNJJ1684.

Institutional Review Board Statement: Not applicable.

Data Availability Statement: All data needed to evaluate the conclusions are present in the paper and/or the supplementary information. All other relevant data are available from the authors upon reasonable request.

Acknowledgments: J. Song gives his personal thanks to Prof. D.E. Morse and R. Levenson (MCDB, UCSB), for their devoted guidance from 2016 to 2018.

Conflicts of Interest: The authors declare no conflict of interest.

\section{References}

1. Groß, A.; Hashimoto, C.; Sticht, H.; Eichler, J.J.F.i.b.; biotechnology. Synthetic peptides as protein mimics. $2016,3,211$.

2. Zelzer, M.; Ulijn, R.V.J.C.S.R. Next-generation peptide nanomaterials: molecular networks, interfaces and supramolecular functionality. Chemical Society Reviews 2010, 39, 3351-3357.

3. Acar, H.; Srivastava, S.; Chung, E.J.; Schnorenberg, M.R.; Barrett, J.C.; LaBelle, J.L.; Tirrell, M. Self-assembling peptide-based building blocks in medical applications. Adv Drug Deliv Rev 2017, 110-111, 65-79, doi:10.1016/j.addr.2016.08.006.

4. Mandal, D.; Shirazi, A.N.; Parang, K.J.O.; chemistry, b. Self-assembly of peptides to nanostructures. 2014, 12, $3544-3561$.

5. Field, L.D.; Delehanty, J.B.; Chen, Y.; Medintz, I.L.J.A.o.c.r. Peptides for specifically targeting nanoparticles to cellular organelles: quo vadis? Accounts of chemical research 2015, 48, 1380-1390.

6. Wang, H.; Feng, Z.; Xu, B.J.A.C. Assemblies of peptides in a complex environment and their applications. Angewandte Chemie 2019, 131, 10532-10541. 
7. Jean, S.R.; Ahmed, M.; Lei, E.K.; Wisnovsky, S.P.; Kelley, S.O.J.A.o.c.r. Peptide-mediated delivery of chemical probes and therapeutics to mitochondria. Accounts of chemical research 2016, 49, 1893-1902.

8. Szeto, H.H.; Liu, S.; Soong, Y.; Wu, D.; Darrah, S.F.; Cheng, F.-Y.; Zhao, Z.; Ganger, M.; Tow, C.Y.; Seshan, S.V.J.J.o.t.A.S.o.N. Mitochondria-targeted peptide accelerates ATP recovery and reduces ischemic kidney injury. Journal of the American Society of Nephrology 2011, 22, 1041-1052.

9. Beyer, H.M.; Juillot, S.; Herbst, K.; Samodelov, S.L.; Muller, K.; Schamel, W.W.; Romer, W.; Schafer, E.; Nagy, F.; Strahle, U.; et al. Red Light-Regulated Reversible Nuclear Localization of Proteins in Mammalian Cells and Zebrafish. ACS Synth Biol 2015, 4, 951-958, doi:10.1021/acssynbio.5b00004.

10. Yumerefendi, H.; Dickinson, D.J.; Wang, H.; Zimmerman, S.P.; Bear, J.E.; Goldstein, B.; Hahn, K.; Kuhlman, B.J.P.o. Control of protein activity and cell fate specification via light-mediated nuclear translocation. 2015, 10, e0128443.

11. Itzhak, D.N.; Tyanova, S.; Cox, J.; Borner, G.H.J.e. Global, quantitative and dynamic mapping of protein subcellular localization. 2016, 5, e16950.

12. Guntas, G.; Hallett, R.A.; Zimmerman, S.P.; Williams, T.; Yumerefendi, H.; Bear, J.E.; Kuhlman, B.J.P.o.t.N.A.o.S. Engineering an improved light-induced dimer (iLID) for controlling the localization and activity of signaling proteins. Proceedings of the National Academy of Sciences 2015, 112, 112-117.

13. Niopek, D.; Benzinger, D.; Roensch, J.; Draebing, T.; Wehler, P.; Eils, R.; Di Ventura, B.J.N.c. Engineering light-inducible nuclear localization signals for precise spatiotemporal control of protein dynamics in living cells. Nature communications 2014, 5, 1-11.

14. Slootweg, E.; Roosien, J.; Spiridon, L.N.; Petrescu, A.-J.; Tameling, W.; Joosten, M.; Pomp, R.; van Schaik, C.; Dees, R.; Borst, J.W.J.T.P.C. Nucleocytoplasmic distribution is required for activation of resistance by the potato NB-LRR receptor Rx1 and is balanced by its functional domains. The Plant Cell 2010, 22, 4195-4215.

15. Drake, K.R.; Kang, M.; Kenworthy, A.K.J.P.o. Nucleocytoplasmic distribution and dynamics of the autophagosome marker EGFP-LC3. PloS one 2010, 5, e9806.

16. Christie, M.; Chang, C.-W.; Róna, G.; Smith, K.M.; Stewart, A.G.; Takeda, A.A.; Fontes, M.R.; Stewart, M.; Vértessy, B.G.; Forwood, J.K.J.J.o.m.b. Structural biology and regulation of protein import into the nucleus. J MOL BIOL 2016, 428, $2060-2090$.

17. Grünwald, D.; Singer, R.H.; Rout, M.J.N. Nuclear export dynamics of RNA-protein complexes. Nature 2011, 475, 333-341.

18. Niopek, D.; Wehler, P.; Roensch, J.; Eils, R.; Di Ventura, B.J.N.c. Optogenetic control of nuclear protein export. NAT COMMUN 2016, 7, 1-9.

19. Vogel, P.; Hanswillemenke, A.; Stafforst, T. Switching Protein Localization by Site-Directed RNA Editing under Control of Light. ACS Synth Biol 2017, 6, 1642-1649, doi:10.1021/acssynbio.7b00113.

20. Shi, T.-Q.; Gao, J.; Wang, W.-J.; Wang, K.-F.; Xu, G.-Q.; Huang, H.; Ji, X.-J.J.A.s.b. CRISPR/Cas9-based genome editing in the filamentous fungus Fusarium fujikuroi and its application in strain engineering for gibberellic acid production. ACS Synth Biol 2019, 8, 445-454.

21. Zhang, L.; Wang, L.; Xie, Y.; Wang, P.; Deng, S.; Qin, A.; Zhang, J.; Yu, X.; Zheng, W.; Jiang, X.J.A.C.I.E. Triple - Targeting Delivery of CRISPR/Cas9 To Reduce the Risk of Cardiovascular Diseases. Angew. Chem. Int. Ed. 2019, 58, 12404-12408.

22. Khalil, A.S.; Lu, T.K.; Bashor, C.J.; Ramirez, C.L.; Pyenson, N.C.; Joung, J.K.; Collins, J.J.J.C. A synthetic biology framework for programming eukaryotic transcription functions. Cell 2012, 150, 647-658.

23. Fonseca, J.P.; Bonny, A.R.; Kumar, G.R.; Ng, A.H.; Town, J.; Wu, Q.C.; Aslankoohi, E.; Chen, S.Y.; Dods, G.; Harrigan, P.; et al. A Toolkit for Rapid Modular Construction of Biological Circuits in Mammalian Cells. ACS Synth Biol 2019, 8, 2593-2606, doi:10.1021/acssynbio.9b00322.

24. Lerner, A.M.; Yumerefendi, H.; Goudy, O.J.; Strahl, B.D.; Kuhlman, B.J.A.s.b. Engineering improved photoswitches for the control of nucleocytoplasmic distribution. ACS Synth Biol 2018, 7, 2898-2907.

25. Engelke, H.; Chou, C.; Uprety, R.; Jess, P.; Deiters, A. Control of protein function through optochemical translocation. ACS Synth Biol 2014, 3, 731-736, doi:10.1021/sb400192a. 
26. Allen, M.E.; Zhou, W.; Thangaraj, J.; Kyriakakis, P.; Wu, Y.; Huang, Z.; Ho, P.; Pan, Y.; Limsakul, P.; Xu, X.; et al. An ANDGated Drug and Photoactivatable Cre-loxP System for Spatiotemporal Control in Cell-Based Therapeutics. ACS Synth Biol 2019, 8, 2359-2371, doi:10.1021/acssynbio.9b00175.

27. Aied, A.; Greiser, U.; Pandit, A.; Wang, W. Polymer gene delivery: overcoming the obstacles. Drug Discov Today 2013, 18, 10901098, doi:10.1016/j.drudis.2013.06.014.

28. Kim, B.K.; Kang, H.; Doh, K.O.; Lee, S.H.; Park, J.W.; Lee, S.J.; Lee, T.J. Homodimeric SV40 NLS peptide formed by disulfide bond as enhancer for gene delivery. Bioorg Med Chem Lett 2012, 22, 5415-5418, doi:10.1016/j.bmcl.2012.07.051.

29. Nematollahi, M.H.; Torkzadeh-Mahanai, M.; Pardakhty, A.; Ebrahimi Meimand, H.A.; Asadikaram, G. Ternary complex of plasmid DNA with NLS-Mu-Mu protein and cationic niosome for biocompatible and efficient gene delivery: a comparative study with protamine and lipofectamine. Artif Cells Nanomed Biotechnol 2018, 46, 1781-1791, doi:10.1080/21691401.2017.1392316.

30. Tammam, S.N.; Azzazy, H.M.; Breitinger, H.G.; Lamprecht, A.J.M.p. Chitosan nanoparticles for nuclear targeting: the effect of nanoparticle size and nuclear localization sequence density. MOL PHARMACEUT 2015, 12, 4277-4289.

31. Yang, L.; Jiang, W.; Qiu, L.; Jiang, X.; Zuo, D.; Wang, D.; Yang, L. One pot synthesis of highly luminescent polyethylene glycol anchored carbon dots functionalized with a nuclear localization signal peptide for cell nucleus imaging. Nanoscale 2015, 7, 61046113, doi:10.1039/c5nr01080b.

32. Yang, L.; Wang, Z.; Wang, J.; Jiang, W.; Jiang, X.; Bai, Z.; He, Y.; Jiang, J.; Wang, D.; Yang, L. Doxorubicin conjugated functionalizable carbon dots for nucleus targeted delivery and enhanced therapeutic efficacy. Nanoscale 2016, 8, 6801-6809, doi:10.1039/c6nr00247a.

33. Levenson, R.; Bracken, C.; Sharma, C.; Santos, J.; Arata, C.; Malady, B.; Morse, D.E.J.J.o.B.C. Calibration between trigger and color: Neutralization of a genetically encoded coulombic switch and dynamic arrest precisely tune reflectin assembly. $J$ Biol Chem 2019, 294, 16804-16815.

34. Song, J.; Levenson, R.; Santos, J.; Velazquez, L.; Zhang, F.; Fygenson, D.; Wu, W.; Morse, D.E.J.L. Reflectin Proteins Bind and Reorganize Synthetic Phospholipid Vesicles. Langumir 2020, 36, 2673-2682.

35. T Das, A.; Tenenbaum, L.; Berkhout, B.J.C.g.t. Tet-on systems for doxycycline-inducible gene expression. Current gene therapy 2016, 16, 156-167.

36. Zhou, X.; Vink, M.; Klaver, B.; Berkhout, B.; Das, A.J.G.t. Optimization of the Tet-On system for regulated gene expression through viral evolution. Gene therapy 2006, 13, 1382-1390.

37. Crookes, W.J.; Ding, L.-L.; Huang, Q.L.; Kimbell, J.R.; Horwitz, J.; McFall-Ngai, M.J.J.S. Reflectins: the unusual proteins of squid reflective tissues. Science 2004, 303, 235-238.

38. Izumi, M.; Sweeney, A.M.; DeMartini, D.; Weaver, J.C.; Powers, M.L.; Tao, A.; Silvas, T.V.; Kramer, R.M.; Crookes-Goodson, W.J.; Mäthger, L.M.J.J.o.t.R.S.I. Changes in reflectin protein phosphorylation are associated with dynamic iridescence in squid. 2010, 7, 549-560.

39. Levenson, R.; DeMartini, D.G.; Morse, D.E.J.A.M. Molecular mechanism of reflectin's tunable biophotonic control: Opportunities and limitations for new optoelectronics. APL Mater 2017, 5, 104801.

40. Levenson, R.; Bracken, C.; Bush, N.; Morse, D.E. Cyclable Condensation and Hierarchical Assembly of Metastable Reflectin Proteins, the Drivers of Tunable Biophotonics. J Biol Chem 2016, 291, 4058-4068, doi:10.1074/jbc.M115.686014.

41. DeMartini, D.G.; Izumi, M.; Weaver, A.T.; Pandolfi, E.; Morse, D.E.J.J.o.B.C. Structures, organization, and function of reflectin proteins in dynamically tunable reflective cells. J Biol Chem 2015, 290, 15238-15249.

42. Phan, L.; Ordinario, D.D.; Karshalev, E.; Walkup IV, W.G.; Shenk, M.A.; Gorodetsky, A.A.J.J.o.M.C.C. Infrared invisibility stickers inspired by cephalopods. J. MATER. CHEM. C 2015, 3, 6493-6498.

43. Dennis, P.B.; Singh, K.M.; Vasudev, M.C.; Naik, R.R.; Crookes-Goodson, W.J.J.A.M. Research update: a minimal region of squid reflectin for vapor-induced light scattering. APL MATER 2017, 5, 120701. 
44. Qin, G.; Dennis, P.B.; Zhang, Y.; Hu, X.; Bressner, J.E.; Sun, Z.; Crookes - Goodson, W.J.; Naik, R.R.; Omenetto, F.G.; Kaplan, D.L.J.J.o.P.S.P.B.P.P. Recombinant reflectin - based optical materials. J POLYM SCI POL PHYS 2013, 51, $254-264$.

45. Ordinario, D.D.; Phan, L.; Walkup IV, W.G.; Jocson, J.-M.; Karshalev, E.; Hüsken, N.; Gorodetsky, A.A.J.N.c. Bulk protonic conductivity in a cephalopod structural protein. Nat Chem 2014, 6, 596-602.

46. Phan, L.; Kautz, R.; Leung, E.M.; Naughton, K.L.; Van Dyke, Y.; Gorodetsky, A.A.J.C.o.M. Dynamic materials inspired by cephalopods. Chem Mater 2016, 28, 6804-6816.

47. Yu, C.; Li, Y.; Zhang, X.; Huang, X.; Malyarchuk, V.; Wang, S.; Shi, Y.; Gao, L.; Su, Y.; Zhang, Y.J.P.o.t.N.A.o.S. Adaptive optoelectronic camouflage systems with designs inspired by cephalopod skins. Proc Natl Acad Sci U S A 2014, 111, 12998-13003.

48. Uversky, V.N.J.A.i.c.; science, i. Protein intrinsic disorder-based liquid-liquid phase transitions in biological systems: Complex coacervates and membrane-less organelles. ADV COLLOID INTERFAC 2017, 239, 97-114.

49. Uversky, V.N.J.C.o.i.s.b. Intrinsically disordered proteins in overcrowded milieu: Membrane-less organelles, phase separation, and intrinsic disorder. Curr Opin Struc Biol 2017, 44, 18-30.

50. Guan, Z.; Cai, T.; Liu, Z.; Dou, Y.; Hu, X.; Zhang, P.; Sun, X.; Li, H.; Kuang, Y.; Zhai, Q.; et al. Origin of the Reflectin Gene and Hierarchical Assembly of Its Protein. Curr Biol 2017, 27, 2833-2842.e2836, doi:10.1016/j.cub.2017.07.061.

51. Schindelin, J.; Arganda-Carreras, I.; Frise, E.; Kaynig, V.; Longair, M.; Pietzsch, T.; Preibisch, S.; Rueden, C.; Saalfeld, S.; Schmid, B.J.N.m. Fiji: an open-source platform for biological-image analysis. Nat Methods 2012, 9, 676-682. 